\title{
Polycations XX: New Monodentate Cationic Ligands and Their Coordination with Ruthenium for the Construction of Complexes Expressing Enhanced Interaction with DNA
}

\author{
Leslie Babukutty, Ethan Moskovic, Davina Wadler, Thomas Strekas, and Robert Engel \\ Department of Chemistry and Biochemistry, Queens College, the City University of New York, 65-30 Kissena Boulevard, \\ Flushing, NY 11367, USA \\ Correspondence should be addressed to Robert Engel, robert.engel@qc.cuny.edu
}

Received 3 July 2012; Revised 24 August 2012; Accepted 26 August 2012

Academic Editor: William Setzer

Copyright ( $\odot 2012$ Leslie Babukutty et al. This is an open access article distributed under the Creative Commons Attribution License, which permits unrestricted use, distribution, and reproduction in any medium, provided the original work is properly cited.

Prior investigations from this laboratory concerned with the preparation of new types of organic cations for a variety of biological and nonbiological applications have been extended to the preparation of cation-bearing ligands with nitrogen coordinating sites for use in complexation reactions with ruthenium cores. The syntheses of new cationic ligands as well as ruthenium complexes bearing them are reported here. The introduction of these new types of ligands is intended to provide to the complexes an enhanced ability to interact with DNA, and thereby to have the potential to be enhanced antitumor agents. Preliminary observations of their interactions with DNA are presented.

\section{Introduction}

The potential for ruthenium-centered agents to serve as antitumor pharmaceuticals has been known for some time [1]. It is known that particular complexes of ruthenium that can serve as antitumor agents act by binding to DNA of the tumor cells through the basic sites on a DNA chain (e.g., a guanine site) and crosslinking the DNA chains through linking to a basic site (e.g., another guanine) on another chain, thus preventing cell division and ultimately leading to cell death $[2,3]$. While the bonds between the nitrogenous ligands and the ruthenium center exhibit strength typical of that of coordinate covalent bonds, strengths less than typical of covalent bonds, they exhibit a kinetic stability that allows them to survive to serve as DNA-binding agents [4].

Prior efforts of this laboratory with polycationic salts (topologically referred to as "strings" owing to their flexible linear array of charges within the structure) have demonstrated an ability to bind into the major groove of doubly stranded DNA and cause changes in the conformational nature of the DNA [5-7].
With this concept of DNA interaction in mind and the ability of nitrogenous complexes of ruthenium to interact with DNA, two series of structures have been devised. These structures have been devised with the anticipation that their cationic ligands would enhance the capability of the metal species for interaction with DNA via binding of cationic sites on the ligands with the (anionic) major groove of doubly stranded DNA. The syntheses of the particular ligand types and the construction of model platinum and ruthenium complexes bearing them are presented here, along with initial investigations of the spectral characteristics of the complexes regarding their interactions.

\section{Results and Discussion}

Two types of cation-bearing ligands that have been designed for incorporation into ruthenium-core complexes are considered here. These monodentate cationic ligands are shown in Figure 1, derived from 4,4' -bipyridyl (a), and nicotinic acid (b). 


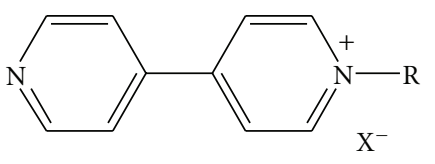

(a)

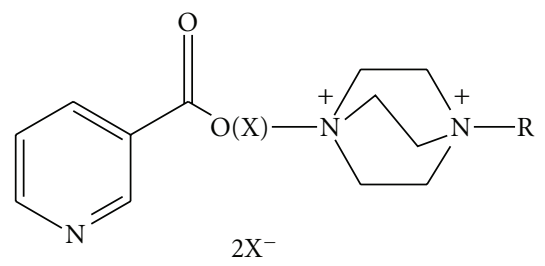

(b)

Figure 1: General structural types of ligands prepared for association with ruthenium core structures: (a) monoalkylated derivatives of 4,4'-bipyridyl; (b) derivatives of nicotinic acid with diazoniabicyclo[2.2.2] octane substituents.

Of the unsubstituted bipyridyls readily available, the $4,4^{\prime}$ bipyridyl is the most conveniently alkylated and results in a monoquaternary salt with the cationic site most fortuitously placed for DNA interaction when complexed with ruthenium. However, alkylation of this material is not as facile as might be anticipated by extension from the chemistry of simple pyridine derivatives. In our hands the only simple alkyl halide that could be used efficiently for the generation of a monoquaternary salt was the 1-bromohexane, which provided the desired salt in low (37.3\%), albeit workable, yield after 21 hours of reflux in ethyl acetate. Other simple alkyl groups could be used to generate monoalkylated $4,4^{\prime}$ bipyridyl derivatives, but in yields too small for efficient use. Prior efforts of our laboratory have indicated that ethyl acetate is a favorable medium for monoalkylation of species bearing several potentially reactive sites, this capability owing to the low solubility of the monoalkylated materials in that solvent $[8-10]$. Further investigation of the solvent systems used is required to perform other related alkylations efficiently.

Facile alkylation for better yield in much shorter reaction time (in ethyl acetate solvent) is accomplished using a variety of benzylic and allylic halides. In these instances, reaction times of under 3 hours of reflux provide reasonable yields of the monoalkylated 4,4'-bipyridyl salts. In all instances, the product salts demonstrated themselves to be significantly hydroscopic, often becoming gummy from collected water during the isolation by filtration. For handling, all of these materials required drying on a vacuum line at $10^{-3}$ Torr prior to use. Even then, significant amounts of moisture were accumulated upon transfer for elemental analyses, and such analyses were performed with the determination of the amount of accumulated water involved. The quaternary ammonium salts (1-8) derived from 4,4'-bipyridyl have been used in the construction of the ruthenium(II) complexes (12-19).

Verification of structure for all isolated salts was performed using NMR in deuterium oxide solution using a $400 \mathrm{MHz}$ Brüker DPX400 instrument with measurement of
${ }^{1} \mathrm{H},{ }^{13} \mathrm{C}$, and ${ }^{13} \mathrm{C}$ Dept 45 (for determination of carbon bearing no hydrogens) spectra, as well as $\mathrm{C} / \mathrm{H} / \mathrm{N}$ elemental analyses (Columbia Analytical Services, Tucson, AZ). These analytical and yield data for the 4,4'-bipyridyl salts are provided in the Experimental section.

There were also generated ligands of general structure (b), specifically ligands noted as (9-10) from unsymmetrically substituted 1,4-diazoniabicyclo[2.2.2] octane salts (11) (vide infra) and 1,3-bis-1'-(4'hexadecyl-1', $4^{\prime}$-diazoniabicyclo[2.2.2] octane)-2-propanol tetrachloride (12) previously reported [11]. These species are derivatives of nicotinic acid and were prepared by reaction of nicotinoyl chloride with the appropriate hydroxyterminal unsymmetrically substituted derivatives of 1,4diazabicyclo[2.2.2] octane. These particular structures of precursors and ligands are shown in Figure 2.

The preparations of the ruthenium complexes using the new cationic ligands were performed using standard procedures. For the ruthenium complexes, a modified approach to the standard procedure [12] was performed by refluxing the hydrate of bis(2,2'-bipyridyl)ruthenium(II) dichloride in aqueous ethanol with the proper equivalent amount of cationic ligand. After hot filtration of the reaction mixture, the filtrate was evaporated under reduced pressure with final traces of ethanol being removed on the vacuum line at $10^{-3}$ Torr.

The preparation of complexes of ruthenium(II) bearing cationic ligands is a relatively simple matter using these approaches. Good-to-excellent yields of the complexes are obtained through these methods, although it is to be noted that all of the complexes are significantly hygroscopic.

Absorbance spectra (UV-Visible) were measured in aqueous solution for the ruthenium complexes prepared. A selection of these spectra is presented here. In several instances (as noted), there appeared slight changes in the spectra after initial preparation of the solutions, but these rapidly came to stability and maintained constant absorption through the UV-Visible region. Absorption spectra for ruthenium complexes 16-18, 20, and 21-22 are shown in Figures 3(a) and 3(b), respectively, with relative maxima and relative intensities noted. Attempts to measure spectra of unhydrated materials failed, as they readily absorbed atmospheric moisture, thus molar absorptivities are provided using the materials for which elemental analyses have been obtained fully hydrated.

Observed absorbances noted represent two types of processes: (1) those of wavelength greater than $320 \mathrm{~nm}$ represent metal-to-ligand charge transfer processes, and (2) those of wavelength less than $320 \mathrm{~nm}$ represent intraligand charge transfer processes.

In several instances, (e.g., ruthenium complexes 16-18) the absorption spectra of initially prepared samples (aqueous solution) underwent shifts of up to $3 \mathrm{~nm}$ in absorption maxima and changes of the values of relative intensities of a few percent. These changes quickly ceased and steady spectra were maintained, observations being made up to a week after initial preparation of the sample solutions. The origin of these small changes was not evident, but can reasonably be accounted for by considering conformational changes in 


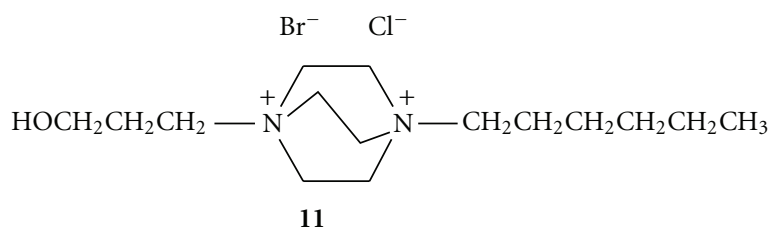

(a)

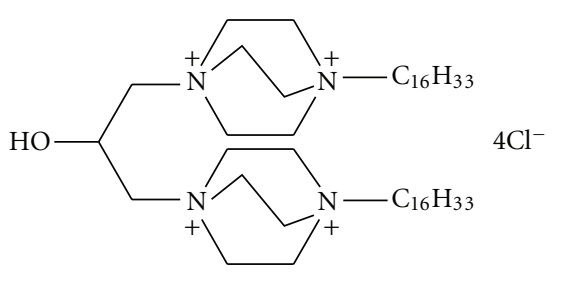

12<smiles>CCCCCC[N+]12CC[N+](CCCOC(=O)c3cccnc3)(CC1)CC2</smiles>

(c)

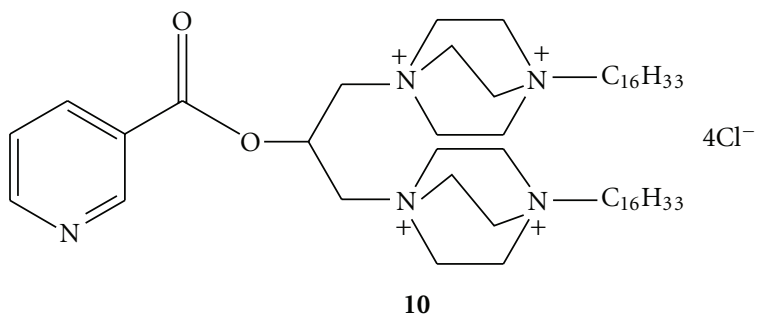

(d)

Figure 2: Precursor and final ligand structures for ligands of category (b) (see Figure 1).

the partially flexible cationic ligands until full equilibrium is attained.

Overall, the observable spectra were typical for octahedral ruthenium with coordination through nitrogen to aromatic ligands [13].

\section{Experimental}

All chemicals and solvents were of reagent quality and were used without further purification as obtained from the supplier. All NMR spectra were measured using a $400 \mathrm{MHz}$ Brüker DPX400 instrument, referenced electronically for both ${ }^{1} \mathrm{H}$ and ${ }^{13} \mathrm{C}$ spectra based on the lock on the deuterated solvent signal. In the instances of the ruthenium complex ${ }^{13} \mathrm{C}$ spectra, resonances resulting from bpy carbon sites are indicated in italics, and carbon sites without hydrogen are indicated by a $q$ after the numerical chemical shift. For these assignments, ${ }^{13} \mathrm{C}$ Dept 45 , HETCOR, and ${ }^{1} \mathrm{H}$ COSY spectra were used in addition to the standard measurements. Elemental analyses were performed for $\mathrm{C} / \mathrm{H} / \mathrm{N} /$ by Columbia Analytical Services of Tucson, AZ, USA.

All UV-visible spectra were measured in distilled water solution using distilled water as a blank, using a HP 8453 diode array UV-visible spectrometer with $1 \mathrm{~cm}$. path quartz cells.

General preparation of monoquaternized 4-substituted-4azonia-4'-azabiphenyl salts (4,4'-bipyridyl salts) (1-8). In a $250 \mathrm{~mL}$ round-bottomed flask is placed $4,4^{\prime}$-bipyridyl ( $10 \mathrm{~g}$,
$0.064 \mathrm{~mol})$ with ethyl acetate $(125 \mathrm{~mL})$ and an equivalent amount of the appropriate organic halide (vide infra) is added to it. The reaction mixture is stirred and heated at reflux for 16 hours, after which time the formed precipitate is recovered by suction filtration through sintered glass, washed with hexane, and placed on the vacuum line at $10^{-3}$ Torr to complete drying. All recovered salts are significantly hydroscopic. The use of simple haloalkanes (such as 1bromohexane) requires longer heating times, albeit with lower isolated yields.

4-Allyl-4-azonia-4'-azabiphenyl chloride (1)-23.4\% yield from allyl chloride- ${ }^{1} \mathrm{H}$ NMR $(\delta)\left(\mathrm{D}_{2} \mathrm{O}\right): 5.16, \mathrm{~d}, 2 \mathrm{H}$; 5.47, m, 2H, 7.74/8.57, $\mathrm{AA}^{\prime} \mathrm{BB}^{\prime}, 4 \mathrm{H} ; 8.29 / 8.83, \mathrm{AA}^{\prime} \mathrm{BB}^{\prime}, 4 \mathrm{H}$. ${ }^{13} \mathrm{C}$ NMR: 63.09, 122.39, 123.20, 125.89, 129.76, 142.32, 144.77, 149.91, 153.82. Calculated for $\mathrm{C}_{13} \mathrm{H}_{13} \mathrm{~N}_{2} \mathrm{Cl} \cdot \mathrm{H}_{2} \mathrm{O}: \mathrm{C}$, 62.28\%; H, 6.03\%; N, 11.17\%. Found: C, 62.03\%; H, 6.10\%; $\mathrm{N}, 11.08 \%$.

4-(2" -methylallyl)-4-azonia-4' -azabiphenyl chloride (2) $-40.3 \%$ yield from 3-chloro-2-methylpropene- $-{ }^{1} \mathrm{H}$ NMR $(\delta)\left(\mathrm{D}_{2} \mathrm{O}\right): 1.66, \mathrm{~s}, 3 \mathrm{H} ; 4.94, \mathrm{~s}, 1 \mathrm{H} ; 5.12$, s, $2 \mathrm{H} ; 5.17$, s, $1 \mathrm{H} ; 7.75 / 8.58, \mathrm{AA}^{\prime} \mathrm{BB}^{\prime}, 4 \mathrm{H} ; 8.29 / 8.86, \mathrm{AA}^{\prime} \mathrm{BB}^{\prime}, 4 \mathrm{H} .{ }^{13} \mathrm{C}$ NMR: 18.62, 61.63, 117.84, 122.38, 125.92, 138.26, 142.16, 144.98, 149.96, 153.83. Calculated for $\mathrm{C}_{14} \mathrm{H}_{15} \mathrm{~N}_{2} \mathrm{Cl} \cdot \mathrm{H}_{2} \mathrm{O}: \mathrm{C}$, 63.51\%; H, 6.47\%; N, 10.58\%. Found: C, 63.40\%; H, 6.61\%; $\mathrm{N}, 10.50 \%$.

4 - $\left(3^{\prime \prime}, 3^{\prime \prime}\right.$-Dimethylallyl)-4-azonia-4' -azabiphenyl chloride (3)-42.0\% yield from 2-Methyl-4-chloro-2-butene${ }^{1} \mathrm{H}$ NMR $(\delta)\left(\mathrm{D}_{2} \mathrm{O}\right): 1.76, \mathrm{~s}, 3 \mathrm{H} ; 1.78, \mathrm{~s}, 3 \mathrm{H} ; 5.13$, 


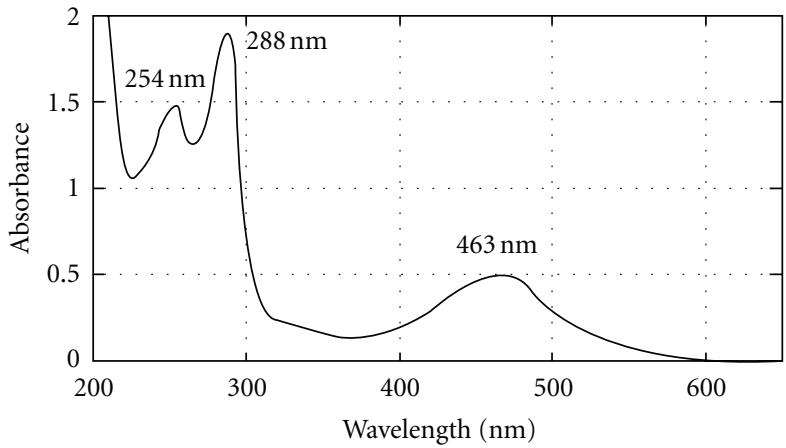

16

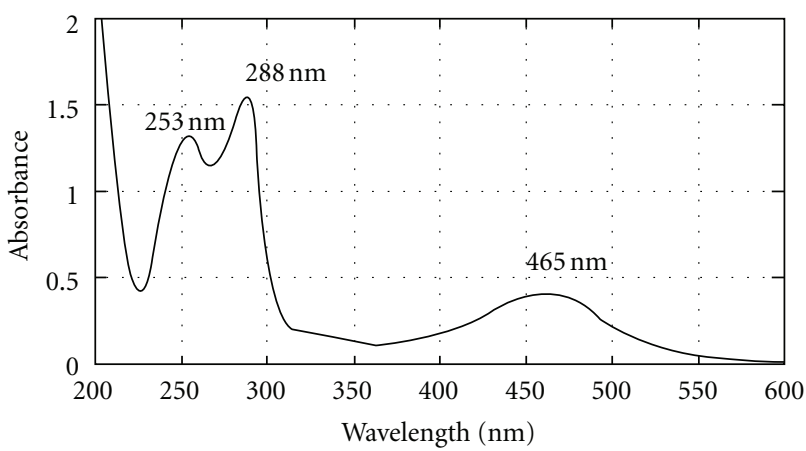

18

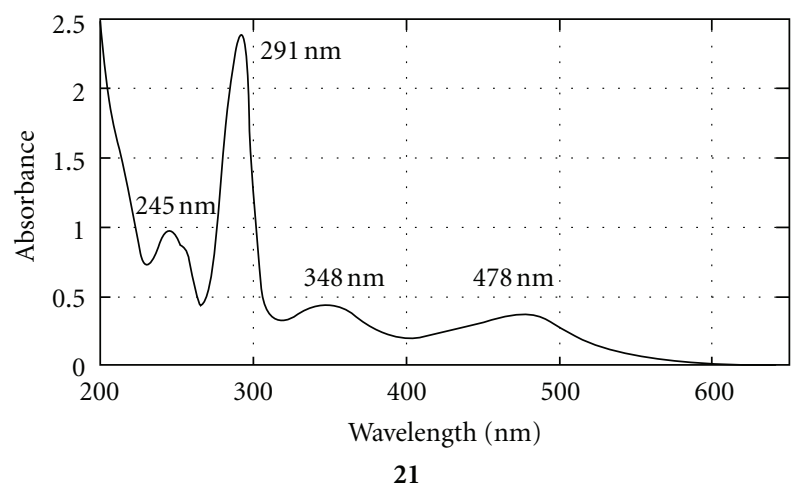

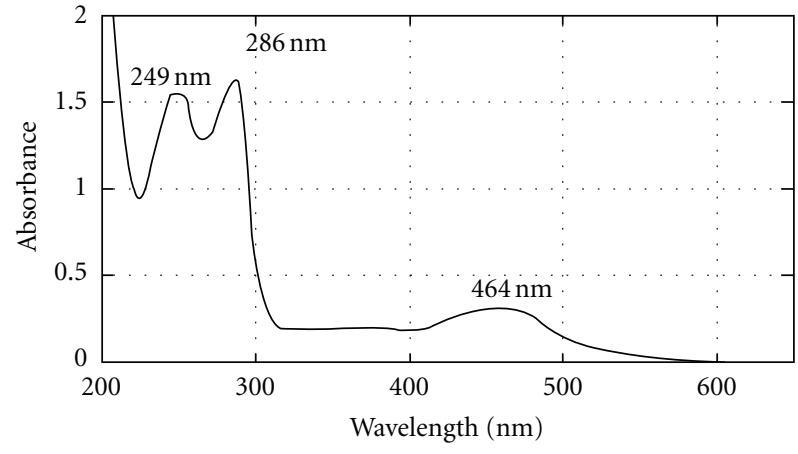

17

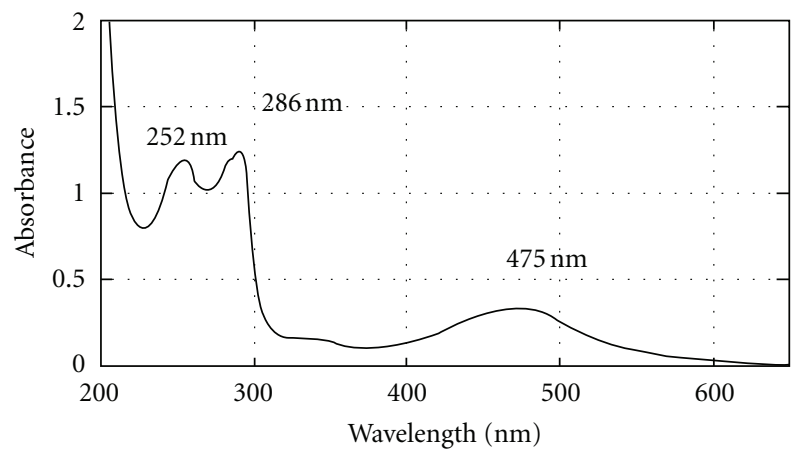

20

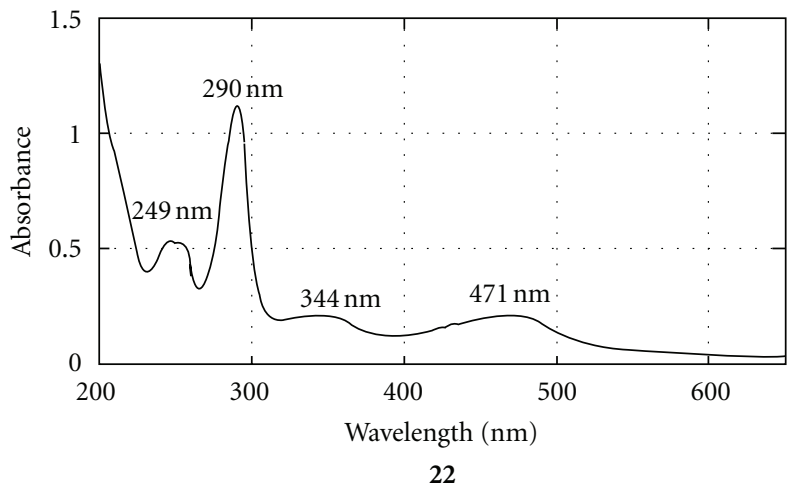

(b)

FIgURE 3: (a) Absorption spectra (200-650 nm) for ruthenium complexes of the type $\mathrm{Ru}(\mathrm{bpy})_{2} \mathrm{~L}_{2}$ for $\mathrm{L}$ being monoalkylated 4,4' -bipyridyl monocations, noted as compounds 16, 17, 18, and 20. Relative maxima and their relative intensities for these spectra are provided in Table 1. (b) Absorption spectra $(200-650 \mathrm{~nm})$ for ruthenium complexes of the type $\mathrm{Ru}(\mathrm{bpy})_{2} \mathrm{~L}_{2}$ for L being esters of nicotinic acid bearing dialkylated diazabicyclo[2.2.2] octane units, noted as compounds $\mathbf{2 1}$ and 22. Relative maxima and their relative intensities for these spectra are provided in Table 1.

br, $2 \mathrm{H} ; 5.46, \mathrm{~m}, 1 \mathrm{H} ; 7.58 / 8.61, \mathrm{AA}^{\prime} \mathrm{BB}^{\prime}, 4 \mathrm{H} ; 8.22 / 8.79$, $\mathrm{AA}^{\prime} \mathrm{BB}^{\prime}, 4 \mathrm{H} .{ }^{13} \mathrm{C}$ NMR: 17.47, 24.94, 61.65, 114.68, 122.40, $125.69,142.54,144.24,145.83,149.82,153.47$. Calculated for $\mathrm{C}_{15} \mathrm{H}_{17} \mathrm{~N}_{2} \mathrm{Cl} \cdot \mathrm{H}_{2} \mathrm{O}$ : C, 64.62\%; H, 6.87\%; N, 10.05\%. Found: C, 64.53\%; H, 7.00\%, N, 9.98\%.

4-Hexyl-4-azonia-4' -azabiphenyl bromide (4)-37.3\% yield from 1-bromohexane- ${ }^{1} \mathrm{H} \operatorname{NMR}(\delta)\left(\mathrm{D}_{2} \mathrm{O}\right): 0.76, \mathrm{t}$, $3 \mathrm{H} ; 1.20, \mathrm{~m}, 6 \mathrm{H} ; 1.96, \mathrm{~m}, 2 \mathrm{H} ; 4.55, \mathrm{t}, 2 \mathrm{H} ; 7.85 / 8.85, \mathrm{AA}^{\prime} \mathrm{BB}^{\prime}$, $4 \mathrm{H} ; 8.32,8.96, \mathrm{AA}^{\prime} \mathrm{BB}^{\prime}, 4 \mathrm{H} .{ }^{13} \mathrm{C}$ NMR: 13.13, 21.68, 24.84, $30.27,30.44,61.64,122.43,125.93,142.55,144.69,149.94$,
153.61. Calculated for $\mathrm{C}_{16} \mathrm{H}_{21} \mathrm{~N}_{2} \mathrm{Br}$ : C, 59.94\%; H, 6.85\%; N, 8.65\%. Found: C, 59.81\%; H, 6.58\%; N, 8.72\%.

4-( $p$-Nitrobenzyl)-4-azonia- ${ }^{\prime}$-azabiphenyl bromide (5) $-79.2 \%$ yield from $p$-nitrobenzyl bromide $-{ }^{1} \mathrm{H}$ NMR ( $)\left(\mathrm{D}_{2} \mathrm{O}\right): 5.89, \mathrm{~s}, 2 \mathrm{H} ; 7.53 / 8.32, \mathrm{AA}^{\prime} \mathrm{BB}^{\prime}, 4 \mathrm{H} ; 7.76 / 8.13$, $\mathrm{AA}^{\prime} \mathrm{BB}^{\prime}, 4 \mathrm{H} ; 8.46 / 8.94, \mathrm{AA}^{\prime} \mathrm{BB}^{\prime}, 4 \mathrm{H} .{ }^{13} \mathrm{C}$ NMR: 63.01, $122.31,123.18,124.45,129.78,139.65,142.26,145.18$, $148.14,149.84,154.44$. Calculated for $\mathrm{C}_{17} \mathrm{H}_{14} \mathrm{~N}_{3} \mathrm{O}_{2} \mathrm{Br}$ : C, 50.76\%, H, 3.51\%; N, 10.45\%. Found: C, 50.69\%; H, 3.63\%; N, $10.27 \%$. 
TABLE 1: Absorption maxima and relative intensities for UV-visible spectra of selected new ruthenium complexes bearing cationic ligands.

\begin{tabular}{|c|c|c|}
\hline Compound & $\lambda_{\max }$ & $\varepsilon\left(\times 10^{4}\right)$ \\
\hline \multirow{3}{*}{16} & 254 & 6.7 \\
\hline & 288 & 8.7 \\
\hline & 463 & 2.3 \\
\hline \multirow{3}{*}{17} & 249 & 12 \\
\hline & 286 & 13 \\
\hline & 464 & 2.4 \\
\hline \multirow{3}{*}{18} & 253 & 11. \\
\hline & 288 & 13. \\
\hline & 465 & 3.5 \\
\hline \multirow{3}{*}{20} & 252 & 5.7 \\
\hline & 286 & 6.0 \\
\hline & 475 & 1.6 \\
\hline \multirow{4}{*}{21} & 245 & 4.2 \\
\hline & 291 & 10. \\
\hline & 348 & 1.8 \\
\hline & 478 & 1.6 \\
\hline \multirow{4}{*}{22} & 249 & 4.1 \\
\hline & 290 & 8.7 \\
\hline & 344 & 1.6 \\
\hline & 471 & 1.6 \\
\hline
\end{tabular}

4-( $p$-Bromobenzyl)-4-azonia-4' -azabiphenyl bromide (6) $-86.1 \%$ yield from $p$-bromobenzyl bromide $-{ }^{1} \mathrm{H}$ NMR ( $\delta)\left(\mathrm{D}_{2} \mathrm{O}\right): 5.76, \mathrm{~s}, 2 \mathrm{H} ; 7.63 / 8.04, \mathrm{AA}^{\prime} \mathrm{BB}^{\prime}, 4 \mathrm{H} ; 7.68 / 8.82$, $\mathrm{AA}^{\prime} \mathrm{BB}^{\prime}, 4 \mathrm{H} ; 8.68 / 9.43, \mathrm{AA}^{\prime} \mathrm{BB}^{\prime}, 4 \mathrm{H} .{ }^{13} \mathrm{C}$ NMR: 61.85, $121.97,122.90,125.87,131.21,132.11,133.61,140.78$, 145.33, 150.94, 152.77. Calculated for $\mathrm{C}_{17} \mathrm{H}_{14} \mathrm{~N}_{2} \mathrm{Br}_{2} \cdot \mathrm{H}_{2} \mathrm{O}: \mathrm{C}$, 46.18\%; H, 4.10\%; N, 6.33\%. Found: C, 45.97\%; H, 3.97\%; $\mathrm{N}, 6.02 \%$.

4-( $p$-Methoxybenzyl)-4-azonia-4' -azabiphenyl chloride (7) $-74.0 \%$ yield from $p$-methoxybenzyl chloride $-{ }^{1} \mathrm{H}$ $\operatorname{NMR}(\delta)\left(\mathrm{D}_{2} \mathrm{O}\right): 3.65, \mathrm{~s}, 3 \mathrm{H} ; 5.61, \mathrm{~s}, 2 \mathrm{H} ; 6.87 / 8.21$, $\mathrm{AA}^{\prime} \mathrm{BB}^{\prime}, 4 \mathrm{H} ; 7.33 / 7.70, \mathrm{AA}^{\prime} \mathrm{BB}^{\prime}, 4 \mathrm{H} ; 7.33 / 8.84, \mathrm{AA}^{\prime} \mathrm{BB}^{\prime}, 4 \mathrm{H}$. ${ }^{13} \mathrm{C}$ NMR: $55.30,61.62,114.84,122.32,124.87,125.89$, $130.97,142.16,144.44,149.89,153.74,159.96$. Calculated for $\mathrm{C}_{18} \mathrm{H}_{17} \mathrm{~N}_{2} \mathrm{OCl} \cdot \mathrm{H}_{2} \mathrm{O}$ : C, 66.35\%; H, 5.79\%; N, 8.47\%. Found: C, $65.29 \%$; H, 5.83\%; N, 8.30\%.

4-( $m$-Methoxybenzyl)-4-azonia-4' -azabiphenyl chloride (8) $-30.4 \%$ yield from $m$-methoxybenzyl chloride $-{ }^{1} \mathrm{H}$ NMR $(\delta)\left(\mathrm{D}_{2} \mathrm{O}\right): 3.67, \mathrm{~s}, 3 \mathrm{H} ; 5.63, \mathrm{~s}, 2 \mathrm{H} ; 6.92-7.34, \mathrm{~m}, 4 \mathrm{H}$; 7.66/8.82, $\mathrm{AA}^{\prime} \mathrm{BB}^{\prime}, 4 \mathrm{H} ; 8.18 / 8.56, \mathrm{AA}^{\prime} \mathrm{BB}^{\prime}, 4 \mathrm{H} .{ }^{13} \mathrm{C}$ NMR: $55.34,63.97,114.57,115.38,121.56,121.58,125.85,130.93$, $133.99,141.95,145.21,149.25,149.89,153.81$. Calculated for $\mathrm{C}_{18} \mathrm{H}_{17} \mathrm{~N}_{2} \mathrm{OCl} \cdot \mathrm{H}_{2} \mathrm{O}$ : C, 66.35\%; H, 5.79\%; N, 8.47\%. Found: C, $65.09 \%$; H, 5.86\%; N, 8.19\%.

Preparation of 3-(1', $4^{\prime}$-Diazonia-4'-hexylbicylco[2.2.2] octyl)-1-propyl nicotinoate bromide chloride (9). In a $100 \mathrm{~mL}$ round-bottomed flask is placed $11(2.09 \mathrm{~g}, 0.0056 \mathrm{~mol})$ with acetonitrile $(50 \mathrm{~mL})$. Nicotinoyl chloride hydrochloride $(1.00 \mathrm{~g}, 0.0056 \mathrm{~mol})$ is added to it, and the resultant mixture is heated at reflux for 24 hours. After cooling, a solution of sodium carbonate hydrate $(1.00 \mathrm{~g}, 0.0081 \mathrm{~mol})$ in water $(5 \mathrm{~mL})$ is added with stirring. After filtering through sintered glass, the filtrate is evaporated under reduced pressure and finally on the vacuum line at $10^{-3}$ Torr to yield a glassy material $(1.74 \mathrm{~g}, 60.6 \%$ yield) that resisted crystallization but provided analytical data in accord with the proposed structure. ${ }^{1} \mathrm{H}$ NMR $(\delta)\left(\mathrm{D}_{2} \mathrm{O}\right): 0.75, \mathrm{t}, 3 \mathrm{H} ; 1.26$, br, $6 \mathrm{H}$; 1.73 , br, $2 \mathrm{H} ; 2.00$, br, $2 \mathrm{H} ; 3.52$, br, $2 \mathrm{H} ; 3.58$, br, $4 \mathrm{H}$; 3.95, br, $12 \mathrm{H}$; 7.51-8.95, m, 4H. ${ }^{13} \mathrm{C}$ NMR: 12.24, 20.45, 20.70, 23.55, 23.91, 33.04, 50.09, 56.78, 57.45, 61.88, 64.35, 137.41, 138.15, 146.88, 148.14, 148.59, 171.12. Calculated for $\mathrm{C}_{21} \mathrm{H}_{35} \mathrm{~N}_{3} \mathrm{O}_{2} \mathrm{BrCl} \cdot 2 \mathrm{H}_{2} \mathrm{O}$ : C, 49.18\%; H, 7.66\%; N, $8.19 \%$. Found: C, 49.07\%, H, 7.81\%, N, 8.11\%.

Preparation of 1,3-bis $\left(1^{\prime}, 4^{\prime}\right.$-diazonia-4'-hexadecylbicyclo[2.2.2] octyl)-2-propyl nicotinoate tetrachloride (10). In a $100 \mathrm{~mL}$ round-bottomed flask is placed 1,3 -bis- $\left(1^{\prime}, 4^{\prime}\right.$ diazonia- $4^{\prime}$-hexadecylbicyclo[2.2.2] octyl)-2-propanol tetrachloride [11] ( $8.54 \mathrm{~g}, 0.0098 \mathrm{~mol})$ with acetonitrile $(60 \mathrm{~mL})$. Nicotinoyl chloride $(1.74 \mathrm{~g}, 0.0098 \mathrm{~mol})$ is added to it, and the reaction mixture stirred for $21 \mathrm{hr}$. At this time, a solution of sodium carbonate hydrate $(1.61 \mathrm{~g}, 0.013 \mathrm{~mol})$ in water $(5 \mathrm{~mL})$ is added with stirring. After filtering through sintered glass, the filtrate is evaporated under reduced pressure and finally on the vacuum line $\left(10^{-3}\right.$ Torr $)$. In this way could be isolated $8.34 \mathrm{~g}(86.9 \%$ yield) of the pure target material. ${ }^{1} \mathrm{H}$ NMR (DMSO- $d_{6}$ ): 0.84, t, 6; 1.25, br, $52 \mathrm{H} ; 1.70$, br, $4 \mathrm{H} ; 3.55-4.00, \mathrm{~m}, 33 \mathrm{H}$; 7.85-9.17, m, 4H. ${ }^{13} \mathrm{C}$ NMR: $13.93,17.94,19.81,19.97,20.88,21.28,22.06,24.26,28.43$, 28.67, 28.72, 28.91, 29.02, 31.26, 38.86, 42.78, 50.14, 51.68, $63.24,65.02,125.41,128.13,140.80,146.91,150.85,164.97$. Calculated for $\mathrm{C}_{53} \mathrm{H}_{99} \mathrm{~N}_{5} \mathrm{O}_{2} \mathrm{Cl}_{4}$ : C, $64.97 \%, \mathrm{H}, 10.18 \% ; \mathrm{N}$, 7.14\%. Found: C, $64.88 \%, \mathrm{H}, 10.22 \%, \mathrm{~N}, 7.07 \%$.

Preparation of 1-hexyl-4-(3'-Hydroxypropyl)-1,4-diazoniabicyclo[2.2.2]-octane chloride bromide (11). In a $100 \mathrm{~mL}$ round-bottomed flask is placed 1-hexyl-1-azonia-4-azabicyclo[2.2.2] octane bromide [10, 11] $(2.04 \mathrm{~g}, 0.0074 \mathrm{~mol})$ with acetonitrile $(40 \mathrm{~mL})$. To this solution is added in excess 3 -chloro-1-propanol $(5.50 \mathrm{~g}, 0.058 \mathrm{~mol})$, and the mixture heated at reflux for 5 hours. After this time, the volatile materials are evaporated under reduced pressure and the gummy residue washed with three portions of ethyl acetate ( $40 \mathrm{~mL}$ each) and the solid dried on the vacuum line at $10^{-3}$ Torr. In this way could be isolated $6.22 \mathrm{~g}(71.9 \%$ yield $)$ of the pure target material. ${ }^{1} \mathrm{H} \operatorname{NMR}(\delta)\left(\mathrm{D}_{2} \mathrm{O}\right): 0.78, \mathrm{t}$, $3 \mathrm{H} ; 1.27, \mathrm{~m}, 6 \mathrm{H} ; 1.73, \mathrm{~m}, 2 \mathrm{H} ; 2.01, \mathrm{~m}, 2 \mathrm{H} ; 3.49-3.67, \mathrm{~m}$, $6 \mathrm{H} ; 3.95, \mathrm{~m}, 12 \mathrm{H} .{ }^{13} \mathrm{C}$ NMR: 12.25, 20.46, 20.72, 23.55, 23.93, 29.37, 50.11, 50.32, 56.79, 57.48, 64.39. Calculated for $\mathrm{C}_{15} \mathrm{H}_{32} \mathrm{~N}_{2} \mathrm{OBrCl} \cdot \mathrm{H}_{2} \mathrm{O}$ : C, $40.59 \% ; \mathrm{H}, 8.99 \%$; N, $6.31 \%$. Found: 40.91\%; H, 9.18\%; N, 6.00\%.

General preparation of new $R u(b p y)_{2}$ complexes (1320). In a $100 \mathrm{~mL}$ round-bottomed flask is placed cisBis-(2,2' -bipyridine) dichlororuthenium(II) hydrate (1.00 g, $0.0020 \mathrm{~mol}$ ) with $95 \%$ ethanol $(50 \mathrm{~mL})$ and the appropriate ligand to be added. For the monodentate ligands as noted above, the amount added is $0.0040 \mathrm{~mol}$. The reaction mixture is stirred and heated at reflux for $20 \mathrm{hr}$, after which time, the hot reaction mixture is filtered through sintered glass, and the filtrate is evaporated under reduced pressure to remove volatile materials, final evaporation being done 
on the vacuum line with a pressure of $10^{-3}$ Torr. All recovered materials are significantly hydroscopic, and elemental analysis was performed to determine not only the veracity of $\mathrm{C} / \mathrm{H} / \mathrm{N}$ composition but also the degree of hydration associated with the isolated materials.

cis-Bis-(2,2' -bipyridine)-bis-(1"'-aza-1'"'-azonia-1'"'-allyl-4" $4^{\prime \prime \prime}$-bipyridine)ruthenium(II) tetrachloride (13) $86.2 \%$ yield from $1-{ }^{1} \mathrm{H}$ NMR $\left(\mathrm{D}_{2} \mathrm{O}\right)$ : 5.12-5.18, br, $4 \mathrm{H} ; 5.49, \mathrm{~m}, 4 \mathrm{H} ; 6.00, \mathrm{~m}, 2 \mathrm{H} ; 7.09-8.85, \mathrm{~m}, 32 \mathrm{H} .{ }^{13} \mathrm{C}$ NMR: $63.10,122.45,122.88,123.08,123.13,123.33,125.68$, $125.99,129.68,129.78,136.74,141.60 q, 142.35 q, 144.83$, $149.91,150.26 \mathrm{q}, 157.68,157.88,159.22 \mathrm{q}$. Calculated for $\mathrm{C}_{46} \mathrm{H}_{42} \mathrm{~N}_{8} \mathrm{Cl}_{4} \mathrm{Ru} \cdot 4 \mathrm{H}_{2} \mathrm{O}: \mathrm{C}, 54.07 \%$; H, $4.93 \%$; N, $10.97 \%$. Found: C, 53.91\%; H, 5.02\%; N, 10.90\%.

cis-Bis-(2,2' -bipyridine)-bis-(1"'-aza-1'"'-azonia-1"' methylallyl- $4^{\prime \prime}, 4^{\prime \prime \prime}$-bipyridine)ruthenium(II) tetrachloride (14)-85.8\% yield from $2-{ }^{1} \mathrm{H}$ NMR $\left(\mathrm{D}_{2} \mathrm{O}\right): 1.63, \mathrm{~d}, 7.5 \mathrm{~Hz}$, $6 \mathrm{H} ; 4.90, \mathrm{~d}, 7.5 \mathrm{~Hz}, 2 \mathrm{H} ; 5.06-5.15, \mathrm{~m}, 6 \mathrm{H} ; 7.00-9.58, \mathrm{~m}$ 32H. ${ }^{13}$ C NMR: $16.74,57.35,117.71,122.88,123.06,125.75$, $126.03,126.75,127.31,138.19,138.28,141.53 q, 142.42 q$, $144.91,149.92 \mathrm{q}, 151.95,152.17,157.65,157.84,159.17 \mathrm{q}$. Calculated for $\mathrm{C}_{48} \mathrm{H}_{46} \mathrm{~N}_{8} \mathrm{Cl}_{4} \mathrm{Ru} \cdot 5 \mathrm{H}_{2} \mathrm{O}$ : C, $53.99 \%$; $\mathrm{H}, 5.29 \%$; N, 10.49\%. Found: 53.80\%; H, 5.43\%; N, 10.47\%.

cis-Bis-(2,2' -bipyridine)-bis-(1"' -aza-1"' -azonia- $1^{\prime \prime \prime}$ $\left[3^{\prime \prime \prime \prime}, 3^{\prime \prime \prime \prime}\right.$-dimethylallyl]-4", $4^{\prime \prime \prime}$-bipyridine)ruthenium(II) tetrachloride (15)-75.8\% yield from 3- ${ }^{1} \mathrm{H}$ NMR $\left(\mathrm{D}_{2} \mathrm{O}\right)$ : $1.73-1.77,4$ poorly resolved singlets, $12 \mathrm{H} ; 5.11, \mathrm{~m}, 4 \mathrm{H}$; 5.45, m, 2H; 7.10-9.61, m, 32H. ${ }^{13} \mathrm{C}$ NMR: 17.46, 24.92, $58.61,114.69,122.42,123.07,125.44,125.74,126.71$, $127.31,136.16,136.72,137.07,142.58 q, 144.32 q, 146.25 \mathrm{q}$, 149.87, 157.66, 157.71, 157.88, 159.20q. Calculated for $\mathrm{C}_{50} \mathrm{H}_{50} \mathrm{~N}_{8} \mathrm{Cl}_{4} \mathrm{Ru} \cdot 4 \mathrm{H}_{2} \mathrm{O}: \mathrm{C}, 55.71 \% ; \mathrm{H}, 5.42 \% ; \mathrm{N}, 10.40 \%$. Found: C, 55.88\%; H, 5.60\%; N, 10.44\%.

cis-Bis-(2,2' -bipyridine)-bis-(1" -aza-1"' -azonia-1"' - $p$ bromobenzyl- $4^{\prime \prime}, 4^{\prime \prime \prime}$-bipyridine)ruthenium(II) dibromide dichloride (16)-92.4\% yield from 6- ${ }^{1} \mathrm{H}$ NMR $\left(\mathrm{D}_{2} \mathrm{O}\right)$ : 5.93, 2, 4H; 7.34-9.47, m, 40H. ${ }^{13} \mathrm{C}$ NMR: 61.88, 121.98, $122.92,125.74,125.87,126.27,126.75,127.21,127.32$, $131.23,132.10 q, 133.47,135.91,136.43,136.53 q, 140.71$, $145.75,151.39 \mathrm{q}, 152.30,157.57,157.77 \mathrm{q}$. Calculated for $\mathrm{C}_{54} \mathrm{H}_{44} \mathrm{~N}_{8} \mathrm{Cl}_{2} \mathrm{Br}_{2} \mathrm{Ru} \cdot 3 \mathrm{H}_{2} \mathrm{O}$ : C, 54.46\%; H, 4.23\%; N, 9.41\%. Found: C, $54.26 \%$; H, 4.31\%; N, 9.28\%.

cis-Bis-(2,2'-bipyridine)-bis-( $1^{\prime \prime}$-aza- $1^{\prime \prime \prime}$-azonia-

$1^{\prime \prime \prime}$-m-methoxybenzyl-4" $4^{\prime \prime \prime \prime}$-bipyridine)ruthenium(II)

tetrachloride (17)-84.6\% yield from 8- ${ }^{1} \mathrm{H}$ NMR $\left(\mathrm{D}_{2} \mathrm{O}\right)$ : $4.70, \mathrm{~s}, 6 \mathrm{H} ; 5.60, \mathrm{~s}, 4 \mathrm{H} ; 6.79-8.82, \mathrm{~m}, 40 \mathrm{H} .{ }^{13} \mathrm{C}$ NMR: $55.30,57.34,114.53,115.34,121.45,121.53,122.12,122.66$, $125.56,125.93,130.90,133.98 q, 136.88,141.81 q, 144.58$, $144.72,144.96 \mathrm{q}, 149.18,149.44,149.88,151.75,152.92 \mathrm{q}$, 157.76q, 159.45q. Calculated for $\mathrm{C}_{56} \mathrm{H}_{50} \mathrm{~N}_{8} \mathrm{Cl}_{4} \mathrm{O}_{2} \mathrm{Ru} \cdot 5 \mathrm{H}_{2} \mathrm{O}$ : C, 56.05\%; H, 5.04\%; N, 9.33\%. Found: C, 55.99\%, H, $5.23 \%$, N, $9.07 \%$.

cis-Bis-(2,2' -bipyridine)-bis-( $1^{\prime \prime}$-aza- $1^{\prime \prime \prime}$-azonia- $1^{\prime \prime \prime}$ hexyl- $4^{\prime \prime}, 4^{\prime \prime \prime}$-bipyridine)ruthenium(II) dibromide dichloride $(\mathbf{1 8})-76.3 \%$ yield from $4-{ }^{1} \mathrm{H}$ NMR $\left(\mathrm{D}_{2} \mathrm{O}\right)$ : 0.69 , $\mathrm{t}$, $6 \mathrm{H} ; 1.09, \mathrm{~m}, 12 \mathrm{H} ; 1.89, \mathrm{~m}, 4 \mathrm{H} ; 4.51, \mathrm{~m}, 4 \mathrm{H} ; 7.61-8.87, \mathrm{~m}$, $32 \mathrm{H} .{ }^{13} \mathrm{C}$ NMR: $13.17,21.70,24.86,30.29,30.47,61.69$, $122.44,122.92,123.45,123.63,125.66,125.77,125.93$, $126.82,137.23,141.59 q, 142.48 q, 144.70,149.92 q, 151.98$,
153.49, 157.82q. Calculated for $\mathrm{C}_{52} \mathrm{H}_{58} \mathrm{~N}_{8} \mathrm{Cl}_{2} \mathrm{Br}_{2} \mathrm{Ru} \cdot 3 \mathrm{H}_{2} \mathrm{O}$ : C, $52.88 \%$; H, 5.46\%; N, 9.48\%. Found: C, 52.61\%; H, $5.70 \% ; \mathrm{N}, 9.33 \%$.

cis-Bis-(2,2'-bipyridine)-bis-( $1^{\prime \prime}$-aza- $1^{\prime \prime \prime}$-azonia- $1^{\prime \prime \prime}$ - [4nitrobenzyl $]-4^{\prime \prime}, 4^{\prime \prime \prime}$-bipyridine)ruthenium(II)dibromide dichloride (19)—90.5\% yield from 5- ${ }^{1} \mathrm{H}$ NMR $\left(\mathrm{D}_{2} \mathrm{O}\right)$ : 5.90, brs, $4 \mathrm{H} ; 7.51-8.97, \mathrm{~m}, 40 \mathrm{H} .{ }^{13} \mathrm{C}$ NMR: $63.05,122.59$, $122.87,123.33,124.44,124.47,126.05,126.41,126.72$, $127.30,129.80,137.17,139.67,141.28,142.54 q, 145.20 q$, $145.26,149.72,151.96,152.17,157.83 \mathrm{q}$. Calculated for $\mathrm{C}_{54} \mathrm{H}_{44} \mathrm{~N}_{10} \mathrm{O}_{4} \mathrm{Cl}_{2} \mathrm{Br}_{2} \mathrm{Ru} \cdot 3 \mathrm{H}_{2} \mathrm{O}: \mathrm{C}, 50.56 \% ; \mathrm{H}, 3.92 \% ; \mathrm{N}$, $10.92 \%$. Found: C, $50.33 \%$; H, 4.01\%; $10.86 \%$.

cis-Bis-(2,2' -bipyridine)-bis-( $1^{\prime \prime}$-aza- $1^{\prime \prime \prime}$-azonia- $1^{\prime \prime \prime}$ [4-methoxybenzyl]-4" $4^{\prime \prime \prime}$-bipyridine)ruthenium(II) tetrachloride (20)-90.2\% yield from 7- ${ }^{1} \mathrm{H}$ NMR $\left(\mathrm{D}_{2} \mathrm{O}\right)$ : 3.69, brs, $6 \mathrm{H}$; 5.65, brs, $4 \mathrm{H} ; 6.90-8.86$, m, $40 \mathrm{H} .{ }^{13} \mathrm{C}$ NMR: $56.05,63.75,114.88,122.42,122.81,123.08,123.30,123.54$, $123.65,125.66,125.98,126.03,126.70,127.31,130.97$, 131.00q, 137.08q, 137.15, 144.56q, 149.87, 152.19, 160.02q. Calculated for $\mathrm{C}_{56} \mathrm{H}_{50} \mathrm{~N}_{8} \mathrm{Cl}_{4} \mathrm{O}_{2} \mathrm{Ru} \cdot 5 \mathrm{H}_{2} \mathrm{O}$ : C, $56.05 \%$; $\mathrm{H}$, 5.04\%; N, 9.33\%. Found: C, 55.91\%, H, 5.26\%, N, 9.16\%.

cis-Bis-(2,2' -bipyridine)-bis-3-(1', $4^{\prime}$-diazonia- $4^{\prime}$ -

hexylbicyclo[2.2.2] octyl)-1-propyl nicotinoate tetrachloride dibromide (21)-62.6\% yield from 9- ${ }^{1} \mathrm{H}$ NMR $\left(\mathrm{D}_{2} \mathrm{O}\right)$ : $0.75, \mathrm{t}, 6 \mathrm{H} ; 1.08, \mathrm{br}, 4 \mathrm{H} ; 1.26, \mathrm{br}, 12 \mathrm{H}, 3.48-3.62, \mathrm{~m}$, $12 \mathrm{H}, 3.93$, br, $24 \mathrm{H}, 7.06-8.51, \mathrm{~m}, 24 \mathrm{H} .{ }^{13} \mathrm{C}$ NMR: 13.10 , $21.35,21.62,24.42,24.83,30.28,51.01,51.22,57.67$, $62.83, \quad 65.29, \quad 123.19, \quad 123.29, \quad 123.35,123.56,123.77$, $125.30, \quad 126.75, \quad 127.51 q, \quad 129.38 q, \quad 131.65 \mathrm{q}, \quad 136.46$, $136.88,137.80,138.73,139.23,178.76 q$. Calculated for $\mathrm{C}_{62} \mathrm{H}_{86} \mathrm{~N}_{10} \mathrm{O}_{4} \mathrm{Cl}_{4} \mathrm{Br}_{2} \mathrm{Ru} \cdot 5 \mathrm{H}_{2} \mathrm{O}: \mathrm{C}, 49.31 \% ; \mathrm{H}, 6.27 \%$; N, $9.28 \%$. Found: C, $49.27 \%, \mathrm{H}, 6.31 \%, \mathrm{~N}, 9.19 \%$.

cis-Bis-(2,2' -bipyridine)-bis-( $1^{\prime}, 4^{\prime}$-diazonia- $4^{\prime}$-hexadecyllbicyclo[2.2.2] octyl)-2-propyl nicotinoate hexachloride (22) - $76.1 \%$ yield from $10-{ }^{1} \mathrm{H}$ NMR $\left(\mathrm{D}_{2} \mathrm{O}\right): 0.74, \mathrm{t}, 12 \mathrm{H}$; 1.12 , br, $104 \mathrm{H} ; 1.68$, br, $8 \mathrm{H}, 3.29-4.15, \mathrm{~m}, 66 \mathrm{H}, 7.02-9.59$, m, 24H. ${ }^{13}$ C NMR: $13.90,16.74,22.66,22.74,22.79,29.08$, $29.44,29.50,29.56,29.82,29.84,29.91,29.99,30.71,31.95$, $44.10,45.66,51.07,51.38,57.37,123.10,123.34,123.56$, $123.65,125.98,126.78,136.12,136.66 q, 136.97 q, 137.78 \mathrm{q}$, 138.02, 151.97, 152.12, 153.06, 167.48, 179.42q. Calculated for $\mathrm{C}_{126} \mathrm{H}_{214} \mathrm{~N}_{14} \mathrm{O}_{4} \mathrm{Cl}_{6} \mathrm{Ru} \cdot 6 \mathrm{H}_{2} \mathrm{O}$ : C, $62.77 \%$; $\mathrm{H}, 9.45 \%$; , $8.13 \%$. Found: C, $62.61 \%, \mathrm{H}, 6.47 \%$, N, $9.05 \%$.

\section{Acknowledgments}

The authors wish to acknowledge the financial support of this project from grants from the US Army, the Research Enhancement Program of Queens College of CUNY, and the PSC-BHE Research Award Program.

\section{References}

[1] P. Pil and S. J. Lippard, "Cisplatin and related drugs," in Encyclopedia of Cancer, vol. 1, pp. 392-410, Academic Press, San Diego, Calif, USA, 1997, Edited by J. R. Bertino.

[2] C. S. Allardyce and P. J. Dyson, "Ruthenium in medicine: current clinical uses and future prospects," Platinum Metals Review, vol. 45, no. 2, pp. 62-69, 2001. 
[3] K. Collins, T. Jacks, and N. P. Pavletich, "The cell cycle and cancer," Proceedings of the National Academy of Sciences of the United States of America, vol. 94, no. 7, pp. 2776-2778, 1997.

[4] I. Kostova, "Platinum complexes as anticancer agents," Recent Patents on Anti-Cancer Drug Discovery, vol. 1, no. 1, pp. 1-22, 2006.

[5] T. C. Strekas, R. Engel, K. Locknauth, J. Cohen, and J. Fabian, "Polycations. 5. Inducement of $\Psi$-DNA circular dichroism signals for duplex deoxyribonucleotide homopolymers by polycationic strings," Archives of Biochemistry and Biophysics, vol. 364, no. 1, pp. 129-131, 1999.

[6] J. I. Cohen, A. Rusinowski, T. C. Strekas, and R. Engel, "Polycations. 6. Polycationic heterocyclic salts: their synthesis and effect on double-stranded DNA," Heteroatom Chemistry, vol. 10, no. 7, pp. 559-565, 1999.

[7] J. Fabian, T. October, A. Cherestes, and R. Engel, "Polycations: syntheses of polyammonium strings as antibacterial agents," Synlett, vol. 1997, no. 8, pp. 1007-1009, 1997.

[8] J. I. Cohen, V. Shteto, and R. Engel, "Polycations 8: the synthesis of polycationic rings," Synthesis, no. 9, pp. 1263-1268, 2000.

[9] J. I. Cohen and R. Engel, "Polyammonium organic saltssyntheses and properties," Trends in Organic Chemistry, vol. 7, pp. 151-157, 2000.

[10] R. Engel, J. I. Rizzo, C. Rivera et al., "Polycations. 18. The synthesis of polycationic lipid materials based on the diamine 1,4diazabicyclo[2.2.2] octane," Chemistry and Physics of Lipids, vol. 158, no. 1, pp. 61-69, 2009.

[11] M. Thomas, D. Montenegro, A. Castaño et al., "Polycations. 17. Synthesis and properties of polycationic derivatives of carbohydrates," Carbohydrate Research, vol. 344, no. 13, pp. 1620-1627, 2009.

[12] A. A. Farah and W. J. Pietro, "Synthesis and characterization of partially crosslinked poly(N-vinylcarbazole-vinylalcohol) copolymers with polypyridyl $\mathrm{Ru}(\mathrm{II})$ luminophores: potential materials for electroluminescence," Polymer Bulletin, vol. 43, no. 2-3, pp. 135-142, 1999.

[13] A. Juris, V. Balzani, F. Barigelletti, S. Campagna, P. Belser, and A. von Zelewsky, "Ru(II) polypyridine complexes: photophysics, photochemistry, eletrochemistry, and chemiluminescence," Coordination Chemistry Reviews, vol. 84, pp. 85-277, 1988. 


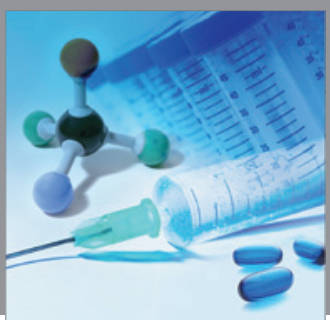

International Journal of

Medicinal Chemistry

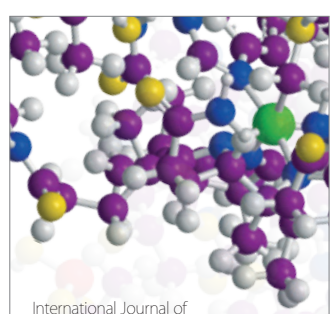

Carbohydrate Chemistry

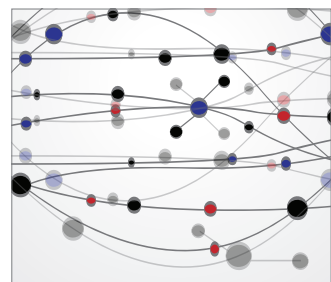

The Scientific World Journal
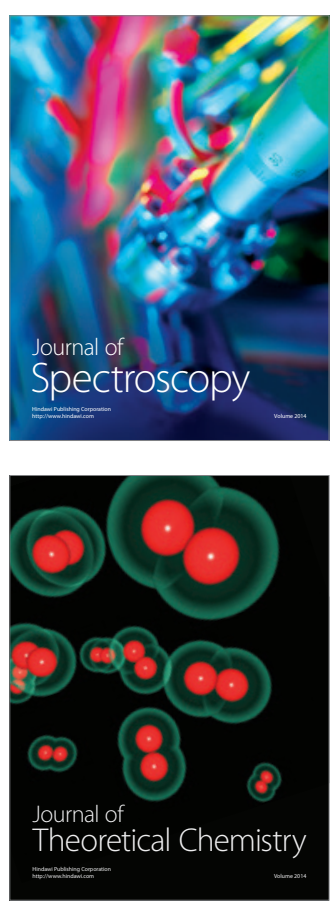
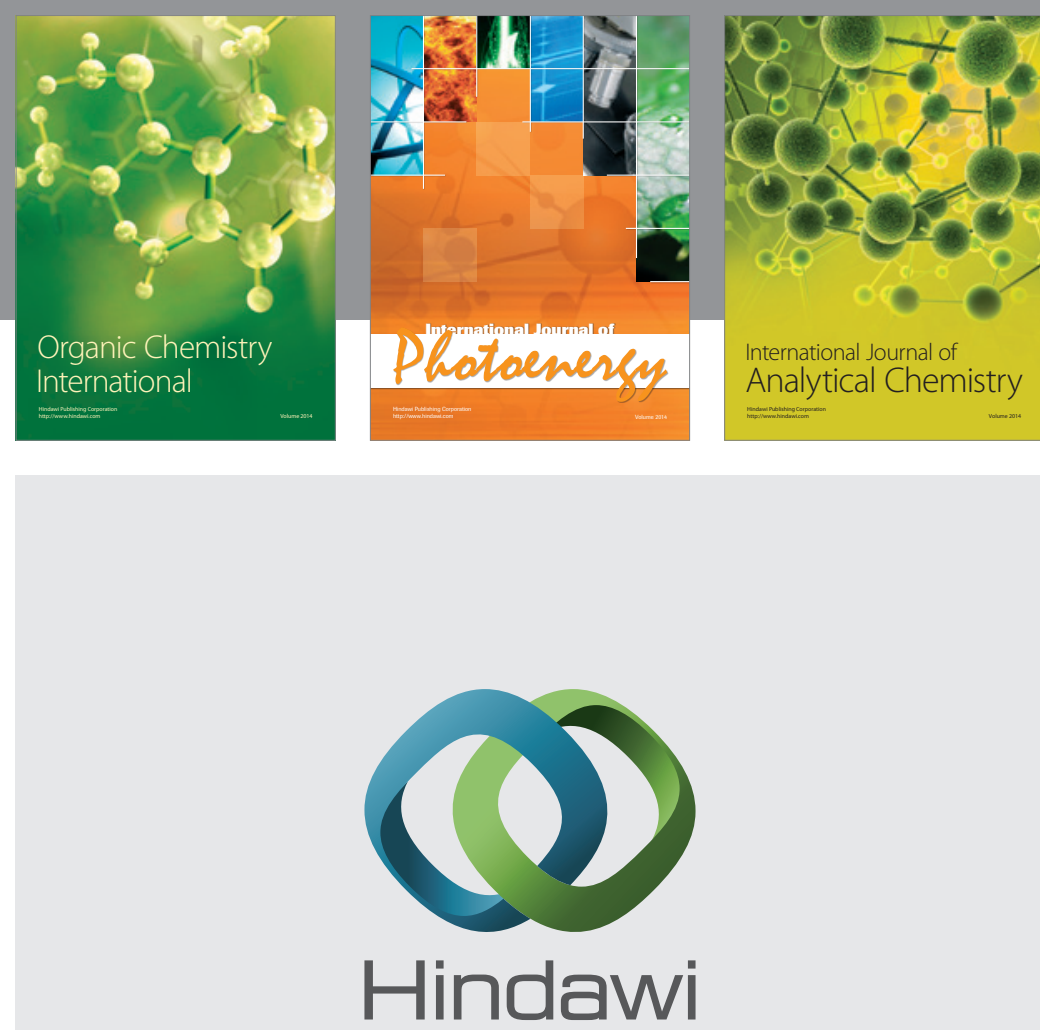

Submit your manuscripts at

http://www.hindawi.com
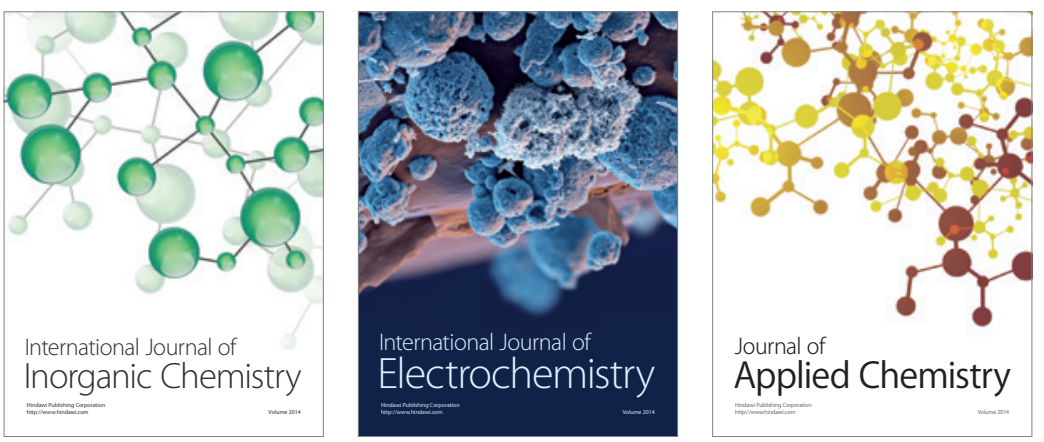

Journal of

Applied Chemistry
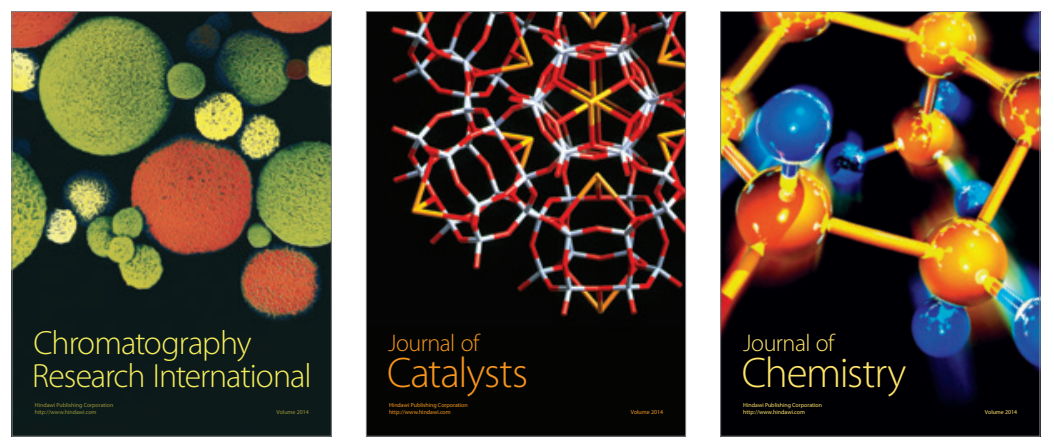
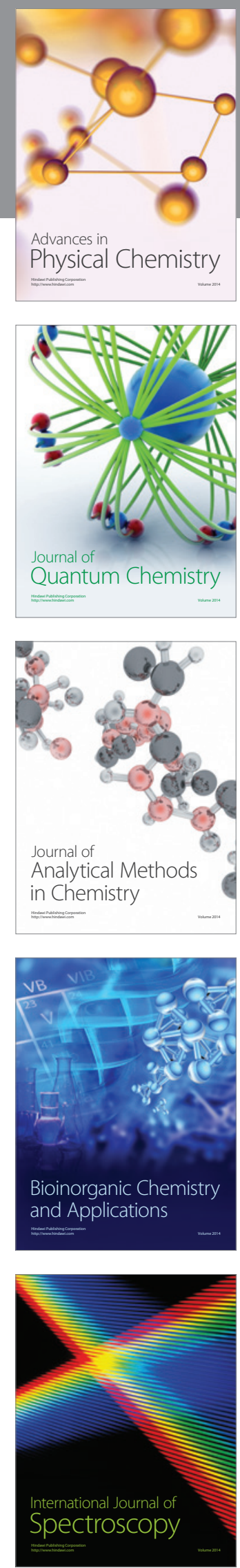